\title{
DRIE-Fabricated Nozzles for Generating Supersonic Flows in Micropropulsion Systems
}

\author{
Robert L. Bayt, Kenneth S. Breuer ${ }^{*}$, and Arturo A. Ayón \\ Massachusetts Institute of Technology/School of Aeronautics and Astronautics \\ 77 Massachusetts Ave 37-401 \\ Cambridge, MA 02139 USA
}

\begin{abstract}
A contoured converging-diverging nozzle has been created for the acceleration of gas flows to supersonic velocities. Extruded two-dimensional devices, with minimum feature sizes averaging 19 microns and 35 microns, are etched using deep reactive ion etching. Mass flow efficiencies through the nozzles range from $88 \%$ to $98 \%$ and are within experimental error to those predicted by a 2-D Navier-Stokes fluid simulation for Reynolds numbers 500 to 4500 . The thrust of a 17.1 to 1 expansion ratio nozzle was measured to be $11.3 \mathrm{mN}$ at 97 psia chamber pressure. This corresponds to an exit velocity of $590 \mathrm{~m} / \mathrm{s}$, which is Mach 3.8 at a chamber temperature of $300 \mathrm{~K}$.
\end{abstract}

\section{INTRODUCTION}

Various trends in the spacecraft industry are driving the development of low-thrust propulsion systems. These may be needed for fine attitude control, or to reduce the mass of the propulsion system through the use of small lightweight components. The nozzle converts the stored energy in the pressurized gas into kinetic energy through an expansion. The nozzle efficiency is characterized by the amount of kinetic energy leaving the nozzle, and is governed by the exit Mach number. In an isentropic nozzle, the exit Mach number is set solely by the ratio of exit area to throat.

Prior to micromachining, low thrust was achieved by fabricating the smallest nozzle possible through conventional machining ( $600 \mu \mathrm{m}$ throat [1]), and then running the device at a low chamber pressure to reduce thrust. However, the device Reynolds number scales with both the nozzle throat width as well as the chamber pressure (or chamber density):

$$
\operatorname{Re}=\frac{\rho a D}{\mu}=\frac{4 \dot{m}}{\pi \mu D}
$$

where $\rho$ is density $a$ is speed of sound, $D$ is width and $\mu$ is viscosity all defined at the throat condition. Mass flow rate (mdot) can be substituted into this expression to yield the term on the right hand side, which is true for an axisymmetric nozzle or a 2-D nozzle of constant aspect ratio. The thrust of a nozzle is approximately equivalent to the momentum flux from the nozzle and can be written as

$$
T \propto \dot{m} u_{e} \propto D \operatorname{Re} u_{e}
$$

where $u_{c}$ is the average exit velocity of the gas. The exit velocity is set by the expansion ratio and remains constant for constant geometries. Thus, if thrust is held constant, Reynolds number increases as feature size decreases for a constant mass flow. Therefore, micromachining can be used to improve nozzle performance over its low-thrust conventionally-machined counterpart by allowing them to be operated at higher Reynolds numbers.

Previous attempts at micromachining supersonic nozzles have been through the anisotropic etching of silicon along crystalline planes using $\mathrm{KOH}$ [2]. This results in symmetric nozzles with a $35.3^{\circ}$ expansion. This is the angle of the flow exiting the nozzle relative to the centerline. This large angle combined with the sharp edge at the throat results in probable flow separation and thus lower mass flow efficiencies than might be achieved in a smoothly varying converging-diverging nozzle. Typical nozzle designs maintain a 15-20 degree expansion and a throat radius of curvature twice that of the throat width. In addition, there is thrust lost to the component of the fluid momentum that is not along the thruster axis, which is larger for high exit divergence angles.

Deep Reactive Ion Etching (DRIE) allows arbitrary geometries to be etched in the plane of the wafer. This affords flexibility in the nozzle geometry that can be used to design against flow separation and minimize divergence losses. By fabricating the nozzle in the wafer plane, the expansion ratio can be made arbitrarily large, where as the $\mathrm{KOH}$ nozzles are limited by the thickness of the wafer. Finally, there is flexibility in the nozzle thrust by adjusting the depth of the etch or the thickness of the wafer. This changes the exit area without changing the expansion ratio and the exit Mach number. However, the nozzle aspect ratio (the ratio of nozzle depth to local width) must be large enough to minimize the influence of the sidewall boundary layers.

\section{DEVICE CONCEPT}

The nozzle-plenum system is illustrated in Figure 1. This isometric view shows the silicon die which has the nozzle pattern etched completely through the chip. The flow channel is encapsulated by anodically bonding Pyrex to the upper and lower surfaces. In order to achieve high device performance, the gas must be injected into the nozzle with as low an entrance velocity as possible. To accomplish this, a settling chamber (or plenum chamber) is fabricated in parallel with the nozzle. This chamber is a region of large volume that is pressurized and remains at nearly constant pressure for the duration of the nozzle firing. The upper surface has been ultransonically drilled with a $1 \mathrm{~mm}$ hole to allow gas injection into the chamber. The nozzle is etched through the wafer in order to maintain a constant feature depth. Partial etches will cause narrow features to etch slower, and result in an uneven nozzle floor. This is further described in the Fabrication section.

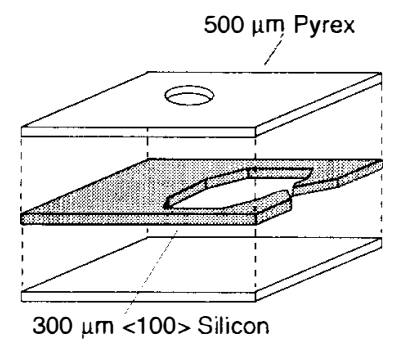

Figure 1: Expanded isometric view of nozzle assembly

\section{FLOW MODELING}

In order to assess the performance of the nozzles, a viscous fluid model is developed that establishes a benchmark to which the experimental work is compared. Due to the nature of the problem,

${ }^{\star}$ Corresponding Author 
flow through a high aspect ratio extruded nozzle, a twodimensional model should adequately simulate the core viscous nozzle flow. The model calculates the steady state conditions through a finite volume simulation of the Navier-Stokes equations. The geometry is non-dimensionalized by the throat width, and the Reynolds number from equation (1) is used to scale the influence of viscosity [3].

The inlet conditions are set by the total pressure in the nozzle chamber, which is assumed to have only an axial component of velocity when entering the nozzle. Since the exit conditions are supersonic except in the boundary layer, the entire exit condition is extrapolated from the interior flow. The sidewalls are considered at a constant temperature consistent with the plenum temperature. Since the thermal mass of the silicon is much larger than that of the gas, the nozzle walls maintain their temperature. Also, the walls are modeled with velocity slip, which is a function of the Knudsen number, the ratio of the mean free path of the fluid to the local feature size. For these runs, the mean free path is sufficiently small that the velocity slip is only important near the region of greatest expansion, and has only a slight effect on the performance of the system ( $1 \%$ variation of thrust at the lowest pressures). Finally, the pressure gradient normal to the wall is a function of the ratio of slip velocity to wall curvature. In regions where curvature is finite, the slip velocity is negligible, and where slip velocity is important the wall is flat. Thus, the wall normal pressure gradient can be neglected, which allows the density to be computed, completing the state vector at the boundaries.

Figure 2 depicts the Mach number distribution through a 5.6:1 area ratio nozzle. This run was performed at a Reynolds number of 1255 that corresponds to a nozzle with a 19-micron throat width at a chamber pressure of 44 psia. The exit Mach number in the simulation is 3.13 , which deviates from the inviscid value of 3.29 . The coefficient of discharge, or mass flow efficiency. is the ratio of the actually mass flow to the theoretical mass flow for a given throat condition. This quantifies the blockage associated with the boundary layers. For this case, the $C_{d}$ is $94.7 \%$.

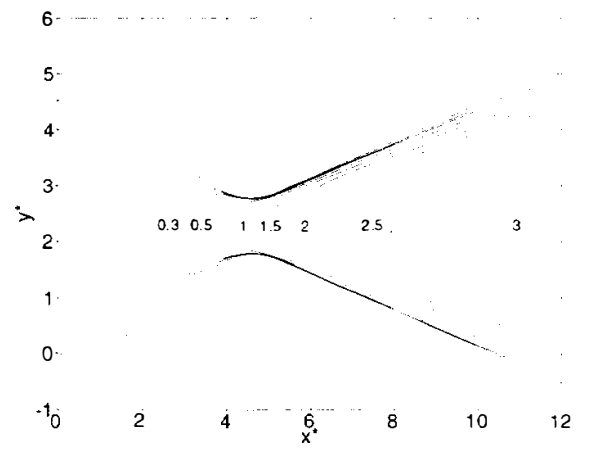

Figure 2: Mach contours for a Reynolds number of 1255 in a nozzle with 5.6:1 expansion ratio. The nozzle axes are not equally dimensioned to ease plot readability.

The model is used to verify the ability of these flow channels to achieve supersonic flow and to determine the performance for various chamber conditions. Three-dimensional effects will reduce the mass flow due to the sidewall boundary layers. A first order calculation predicts a sidewall boundary layer of the same order as those found on the nozzle surfaces in the simulation. This is about 20 microns at the nozzle exit. For a 300-micron deep etch, this is $15 \%$ of the total cross section, which justifies performance estimations made with the 2-D model. The comparison of the simulation's predictions with experimental data is detailed in the Results section.

\section{FABRICATION}

The micronozzle process flow is straightforward in principle. The challenge lies in attaining a highly variable geometry that maintains anisotropy over the full height of the structure. Large features etch faster than small features, which causes the smaller features to distort when performing a deep etch. If small features of a constant geometry are etched, wall roughness becomes accentuated.

At the heart of this research program is a Surface Technology Systems (STS) deep reactive ion etcher [4]. The Multiplex ICP is an etcher that maintains tight control on ion directionality by using an inductively coupled plasma which allows anisotropy to be maintained to great depths.

The process flow is shown in Figure 3. A clean wafer is protected with photoresist that can be applied with thicknesses up to 12 microns. The resist is patterned and developed and the resulting features are etched through the wafer. Typical etch rates are measured between $2-3 \mu \mathrm{m} / \mathrm{min}$ depending on feature size and recipe. The silicon wafers are mounted to quartz wafers with photoresist to prevent any problems when the features etch through the wafer, and allow the cleared features to be detected by inspection.

a)

b)

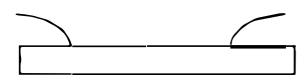

c)

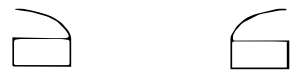

d)

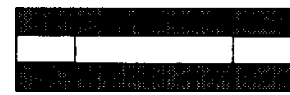

Figure 3: Fabrication sequence for the micronozzle. a) begin with a clean <100> wafer b) coat in photoresist c) DRIE through nozzle 4) Strip resist and anodically bond to glass

The upper surface is anodically bonded [5] to Pyrex at $500^{\circ} \mathrm{C}$ with 1000 Volts and 2400 mbars of pressure applied across the stack. This task is accomplished with an Electronic Visions/Aligner Bonder. The bonder enables the silicon wafer to be aligned with alignment holes in the glass permitting the gas injection holes to line up with the chambers. The Pyrex wafer has an auxiliary hole drilled in it so that electrical contact can be made, through a brass spring, with the silicon when the second Pyrex wafer is bonded to the stack. This contact allows a voltage to be applied only relative to the second Pyrex wafer and the silicon while maintaining the integrity of the first bond. Finally, the wafer is diced along lanes which intersect the nozzle, but prevent the nozzle geometry from being distorted by inaccurate blade alignment. The dicing exposes the flow channel permitting the gas to discharge from the nozzle through the edge of the die. The dicing is accomplished with a resin-bonded blade.

Micrographs of the first attempt at fabrication are depicted in Figure 4a. This process was reported previously by Bayt et al [3]. This process etches the full nozzle-plenum geometry simultaneously. The DRIE performs well for about 200 microns depth with the full geometry. There is noticeable loading effect at the throat, which is designed to have a width of 8 microns. The etch rate is 0.3 $\mu \mathrm{m} /$ minute less than the larger features. Ultimately, the throat region becomes distorted through the over etching that is necessary to clear the throat region. This distortion is obvious near the bottom of the throat in Figure $4 \mathrm{~b}$. This process results in functioning nozzles, but the geometry is distorted and prevents adequate comparison to the fluid model. 
a)

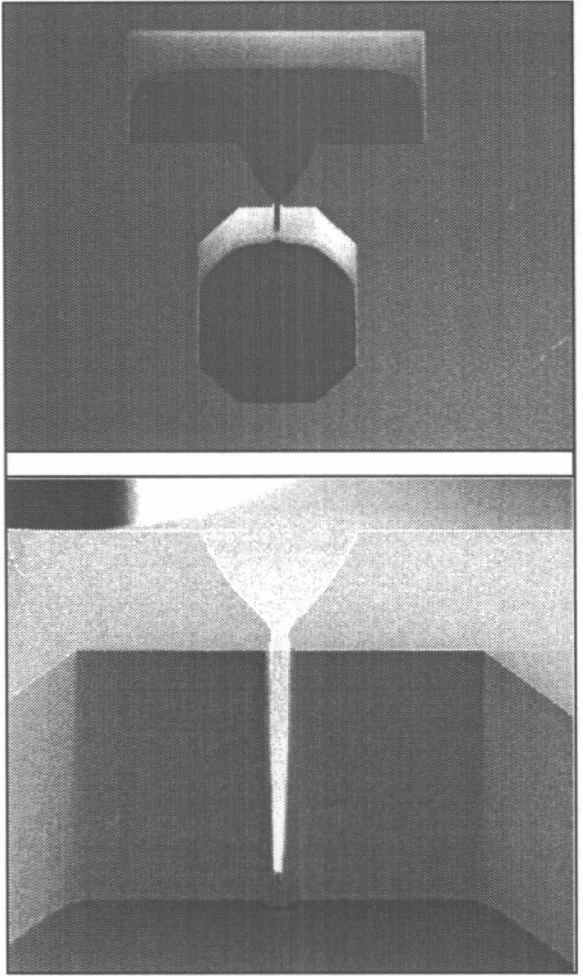

Figure 4: a) Micrograph of a nozzle-plenum system with associated dicing box attached to the nozzle b) close-up of nozzle throat revealing distortion in geometry near bottom of etch

One attempt at reducing the loading effect was to use a nested mask set that allows the nozzles to be etched initially to a depth of about 40 microns before the larger features are exposed. An oxide layer is patterned with the nozzles, and then coated with photoresist, which is patterned with the settling chamber. The STS etcher begins the etch in the nozzle region, and then the oxide is stripped in the settling chamber region with a buffered oxide etch. The wafer then goes back into the STS to complete the etch. The success of this scheme is too constrained by the etch timing, and once again leads to overetching and feature distortion. It is apparent from these results that success would only be possible through etching a constant feature size.

The optimal geometry was eventually achieved by etching a feature of similar size to maintain constant loading. A halo mask accomplishes this. The nozzle outline is defined by a 10-micron trench outlining the feature of interest. This allows the centerbody to fall out after the etch is completely through the wafer. Because of small feature distortion, best results are obtained for etches 300 microns in depth. Figure 5 is a micrograph of a nozzle for which this process was used. The anisotropy of this nozzle is striking. There is only a 1-micron variation in feature size from the top to the bottom of the wafer. The wall roughness is larger for this type of mask due to the small halo trench. The roughness is on the order of 1 micron, where it had been about 0.3 microns with the previous processes.

This etch process results in feature enlargement from the photoresist mask which breaks down over time. The features are 10 microns on the mask and become 18 microns by the completion of the etch. It may be possible to mitigate this affect with an oxide mask. In addition to feature enlargement, there is a variation in etch rate across the wafer due to asymmetric etchant delivery to the chamber. Further characteristics of the STS etcher can be found in Ayon et al [6].

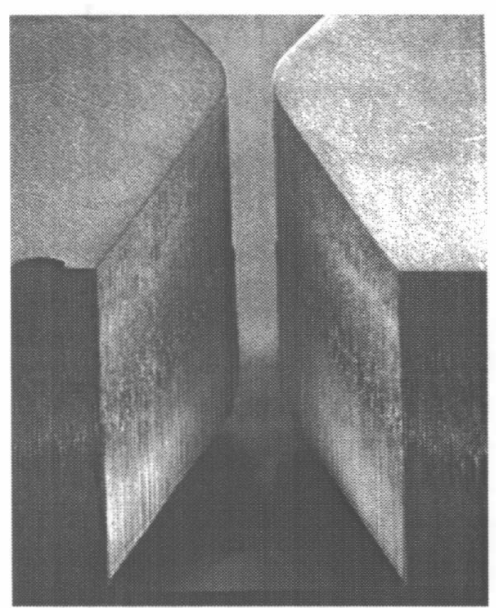

Figure 5: Micrograph of nozzle etched using a halo mask. Throat diameter is 20 micron with and expansion ratio of 5.4:1

Once the die has been fabricated, it must be packaged to interface with the macroscopic world. Figure 6 depicts the gas injection manifold that allows the die to interface with a standard high-pressure source. The manifold consists of an o-ring gland that runs to a $1 / 4$ " pipe fitting that is interconnected with stainless steel tubing. The die is clamped against the o-ring to prevent fluid leakage. A valve and regulator are present upstream to allow flow control to the nozzle. Pressure in the chamber is read from a transducer that is integrated into the manifold. The pressure drop in the flow channels leading to the die is less than $0.1 \mathrm{psi}$ at these low flow rates.

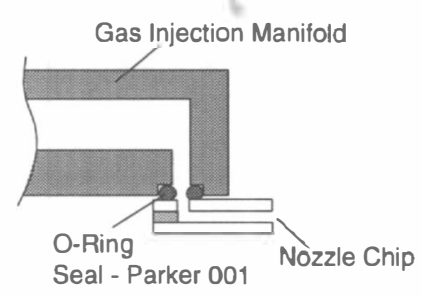

Figure 6: Nozzle Packaging Concept

\section{EXPERIMENTAL RESULTS}

The test set-up utilizes grade 5.0 nitrogen regulated to chamber pressures ranging from 5 to 300 psia. A 0.5 -micron filter is in line to prevent contamination from entering the nozzle. The flow rate is measured using a Teledyne-Hastings HFM-200 0-1000 sccm flow meter which is accurate to $0.5 \%$ of full scale, and the system is verified to be free of leaks before tests are run. The results reported in Bayt et al [3] verifies that sonic flow is achieved in nozzles. Since these results, the more recent mass flow measurements with improved accuracy have produced data that can be compared against the flow model data.

Figure 7 compares the mass flow test data with the model data. At the highest Reynolds number the test data is higher than the model data, but to within experimental uncertainty. In addition to the error in the mass flow meter, the feature geometry can only be measured in plane to within 0.5 microns, which results in a $2.5 \%$ uncertainty in the theoretical mass flow. As the pressure is reduced, and hence the Reynolds number is reduced, the experimental $C_{d}$ falls off much faster than the model data. This is due to the influence of the sidewall boundary layers not modeled in the 2-D simulation. 


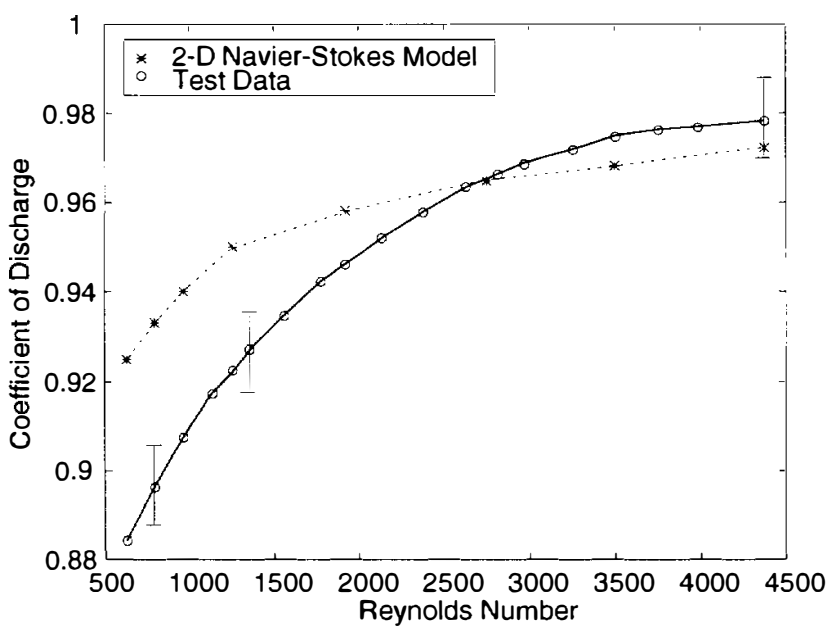

Figure 7: Mass flow results for both the fluid model and the experimental testing for a nozzle with a 19-micron throat and a 5.4:1 expansion ratio

This trend is consistent for the different nozzle geometries tested with similar lengths and throat Reynolds numbers. For nozzles with similar throat Reynolds number but with different expansion ratios (and therefore lengths) the $C_{d}$ is degraded due to the additional boundary layer growth incurred.

Finally, tests were performed with the devices to determine the thrust level. The thrust stand is accurate to 1 milliNewton of thrust over the range of $1-20 \mathrm{mN}$. For a nozzle with a 37 -micron throat and a 17.1:1 expansion ratio, $11.3 \mathrm{mN}$ of thrust was generated at a chamber pressure of 97.2 psia. The mass flow of this device was $924 \mathrm{sccm}$, which results in an exit velocity of $590 \mathrm{~m} / \mathrm{s}$. This is approximately Mach 3.8 as compared to the isentropic value of 4.5 . Thus, the thrust tests verify that supersonic flow has been achieved.

The thrust tests, which are detailed in [7], are shown in Figure 8. The exit velocity is a direct indication of the momentum exchange for this device, thus it is a direct indication of the nozzle performance. The exit velocity should remain insensitive to chamber pressure and thrust for high Reynolds numbers. The decrease in exit velocity (hence performance) is due to the low Reynolds number viscous effects which are present at the low chamber pressures.

\section{CONCLUSIONS}

Supersonic flow can be achieved in micron-scale contoured devices to be used in micropropulsion systems. This is possible because of the anisotropy afforded during nozzle fabrication by deep reactive ion etching. This allows features to be etched which are of a constant geometry between the upper and lower surfaces where the geometric measurements are quantified. Feature distortion occurs when objects of variable geometry are etched. This can be overcome by etching a trench of constant width about the feature of interest. Thrust measurements indicate that Mach 3.8 flow is achieved for 17:1 expansion ratios, which is degraded from the 4.5 predicted by isentropic theory. The mass flow rates of these devices can be measured and produce similar repeatable results for nozzles of similar lengths and Reynolds numbers. The experimental results indicate a reduction in mass flow efficiency with Reynolds numbers. This efficiency compares well with the numerical model at high Reynolds numbers, and deviates at low Reynolds number due to the three-dimensional effects of the flow.

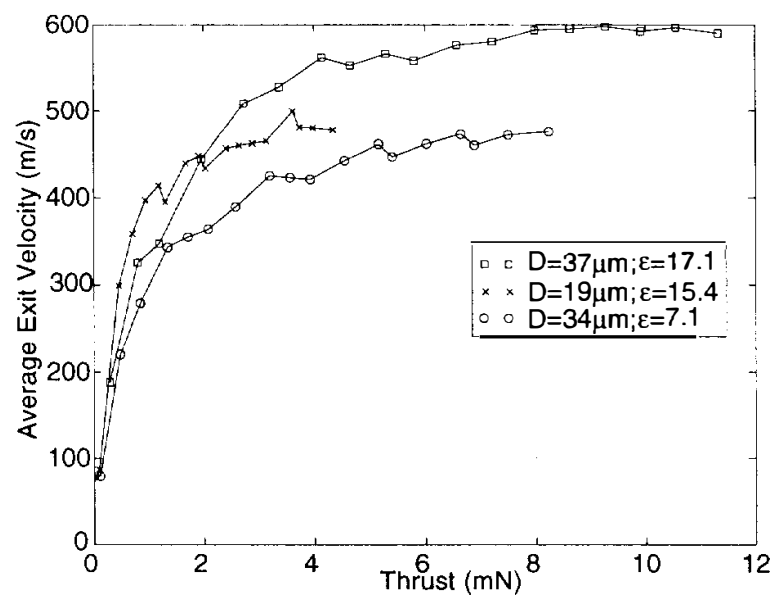

Figure 8: Variation of Exit Velocity with Thrust for several nozzle geometries

\section{ACKNOWLEDGEMENTS}

The authors would like to thank the JPL Microdevices Laboratory by whom this program was funded. In addition, we would like to thank Siegfried Janson at the Aerospace Corporation for the use of their thrust stand for measurements presented here. Also, our gratitude is extended to Professor Martin A. Schmidt of the Microsystems Technology Laboratory for his assistance and insight in the development of the process flow.

\section{REFERENCES}

1. S.P. Grisnik, T.A. Smith, L.E. Salz, 'Experimental Study of Low Reynolds Number Nozzles", AIAA Paper 87-0092, May 1987.

2. S.W. Janson, and H. Helvajian, "Batch-Fabricated Microthrusters: Initial Results", $32^{\text {nd }}$ AIAA Joint Propulsion Conference AIAA Paper 96-2988, July 1996.

3. R.L. Bayt, A.A. Ayon, and K.S. Breuer, "A Performance Evaluation of MEMS-based Micronozzles", $33^{\text {rd }}$ AIAA Joint Propulsion Conference AIAA Paper 97-3169, July 1997.

4. R. Bosch, "Method for anisotropically etching silicon", US Patents 4855017, 4789720, Germany Patent 4241045C1.

5. G. Wallis and D.I. Pomerantz, "Field Assisted GlassMetal Sealing", Journal of Applied Physics, 40, 10, September 1969, pp. 3945-3949.

6. A. Ayon, B. Braff, C. Lin, H. Sawin, M. Schmidt, "Characterization of an Inductively Coupled Plasma Etcher", submitted to the Journal of the Electrochemical Society.

7. R.L. Bayt and K.S. Breuer, "Performance Enhancement to MEMS-Based Mircopropulsion Systems", $34^{\text {th }}$ AIAA Joint Propulsion Conference AIAA Paper 98-3921, July 1998. 